\title{
Bioorthogonal Elastin-like Polypeptide Scaffolds for Immunoassay Enhancement
}

\author{
Duy Tien $\mathrm{Ta}^{1,2}$, Rosario Vanella ${ }^{1,2}$, Michael A. Nash ${ }^{1,2, *}$ \\ ${ }^{1}$ Department of Chemistry, University of Basel, 4058 Basel, Switzerland \\ ${ }^{2}$ Department of Biosystems Science and Engineering, ETH Zurich, 4058 Basel, Switzerland. \\ * Corresponding author: michael.nash@unibas.ch
}

\begin{abstract}
Artificial multiprotein complexes are sought after reagents for biomolecular engineering. A current limiting factor is the paucity of molecular scaffolds which allow for site-specific multi-component assembly. Here, we address this limitation by synthesizing bioorthogonal elastin-like polypeptide (ELP) scaffolds containing periodic non-canonical L-azidohomoalanine amino acids in the guest residue position. The 9 azide ELP guest residues served as conjugation sites for site-specific modification with dibenzocyclooctyne (DBCO)functionalized single-domain antibodies (SdAbs) through strain-promoted alkyne-azide cycloaddition (SPAAC). Sortase A and ybbR tags at the C- and N-termini of the ELP scaffold provided two additional sites for derivatization with small molecules and peptides by Sortase A and 4'-phosphopantetheinyl transferase (Sfp), respectively. These functional groups are chemically bioorthogonal, mutually compatible, and highly efficient, thereby enabling synthesis of multi-antibody ELP complexes in a one-pot reaction. We demonstrate application of this material for enhancing the performance of sandwich immunoassays of the recombinant protein mCherry. In undiluted human plasma, surfaces modified with multi-antibody ELP complexes showed between 2.3 and 14.3-fold improvement in sensitivity and $\sim 30-40 \%$ lower limits of detection as compared with nonspecifically adsorbed antibodies. Dual-labelled multi-antibody ELP complexes were further used for cytometric labeling and analysis of live eukaryotic cells. These results demonstrate how multiple antibodies complexed onto bioorthogonal ELP scaffolds can be used to enhance immunospecific binding interactions through multivalency effects.
\end{abstract}

Key words: ELP, single-domain antibody, SPAAC, protein scaffold, multiprotein complex. 


\section{Introduction}

The synthesis of molecular scaffolds for assembly of multiprotein complexes in a defined configuration has been a long-term goal in the biomolecular engineering field. Many different scaffolds for the assembly of artificial multiprotein complexes have been reported ${ }^{1}$, for example, genetically-fused repeating copies of receptor proteins which serve as docking sites ${ }^{2-9}$, DNA origami ${ }^{10-13}$, or multi-protein assemblies linked together through bioorthogonal conjugation ${ }^{14}$, enzymatic ligation ${ }^{15}$, and isopeptide bond formation ${ }^{16-18}$ to name only a few.

Among the various candidates for scaffold design, elastin-like polypeptides (ELPs) comprising (VPGXG) pentapeptide repeats have several attractive features. Their tunable phase transition characteristics impart an ability to aggregate and precipitate from aqueous solutions at temperatures above the lower critical solution temperature (LCST) ${ }^{19-22}$. Since ELPs are composed exclusively of peptide-bonded residues, they are non-toxic, biocompatible, and have uniform elastic response under force ${ }^{23}$. They are intrinsically disordered linear proteins ${ }^{24}$ but unlike synthetic polymers, are genetically encoded and therefore completely monodisperse and able to self-assemble with cargo molecules ${ }^{25}$. Furthermore, the ability to create block ELP architectures through straightforward cloning procedures is highly advantageous.

The use of ELPs as scaffolds for nanoassembly using standard protein coupling strategies is severely limited by cross-reactivity among naturally occuring amino acid side chains. Introduction of bioorthogonal groups through posttranslational modification of standard amino acids ${ }^{26}$ or through genetic code expansion ${ }^{27-30}$ would be a promising approach ${ }^{31,32}$. Bioorthogonal ELPs were reported by Smits et al. ${ }^{26}$ who installed bioorthogonal azide groups onto the $\varepsilon$-amine of lysine guest residues in ELPs. Petitdemange et al. reported design of a methionine-containing ELP that allowed for post-translational modification (e.g. alkylation) via the methionine guest residues ${ }^{45}$. Teeuwen et al. ${ }^{33}$ expressed an alkyne- or azide- endfunctionalized ELP using a methionine-auxotrophic $E$. coli strain in medium supplemented with homopropargylglycine or azidohomoalanine, respectively. Recent studies ${ }^{34-38}$ have also reported multisite incorporation of bioorthogonal functional groups into ELPs at the guest residue position, however, such approaches have typically relied on custom bacterial strains that enacted specific suppression of standard or amber codons, requiring an orthogonal aminoacyl-tRNA synthetase.

Since ELPs contain only a limited set of amino acids, we hypothesized that sense suppression ${ }^{39,40}$, which relies on the natural promiscuity of $E$. coli Met aminoacyl-tRNA synthetase to charge native tRNAs with a bioorthogonal amino acid, would be a valuable approach for bioorthogonal ELP scaffold engineering at the guest residue position. We therefore sought to implement genetically encoded nonstandard amino acids into repetitive protein polymers and to demonstrate efficient strategies to conjugate multiple antibody molecules onto ELP scaffolds using one-pot parallel bioorthogonal click-like reactions. 


\section{Results and Discussion}

The protein design and strategy for multiprotein complex assembly demonstrated in this study are shown in Fig. 1. We designed the ELP with multiple copies of a starting polypeptide containing one methionine, followed by seven valine and two glutamic acid as guest residues, resulting in a ratio of 1:7:2 for $M, V$ and $E$, respectively $\left(M_{1} V_{7} E_{2}\right)$ (Fig. 1A). Using recursive directional ligation ${ }^{41}$ (Supporting information Fig. S1), we produced an ELP gene encoding in total 90 pentapeptide repeats, 9 of which contained Met codons in the guest position, denoted $\left(\mathrm{M}_{1} \mathrm{~V}_{7} \mathrm{E}_{2}\right)_{9}$ under control of a $T 7 /$ lac promoter. After confirming the sequences, we transformed the plasmids into the E. coli strain RF11, which is a C43(DE3)-based Met-auxotroph ${ }^{42}$ capable of up taking and incorporating the nonstandard amino acid azidohomoalanine (Met analog) into proteins via sense suppression. Expression of the azide-containing ELPs was achieved by growing up a single colony in minimal media supplemented with all 20 natural amino acids. During IPTG induction the cells were transferred into minimal media lacking methionine and supplemented with azidohomoalanine. Cells were grown overnight at room temperature and the resulting ELPs purified using chromatography-free inverse transition cycling (ITC) as described in our previous study ${ }^{20}$.

\section{a ELP design}

ybbR tag

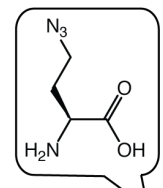

ELP with multi-site guest residue L-azidohomoalanine

DSLEFIASKLA [VPGM ${ }^{\text {azide }}$ G-(VPGVG) $)_{7}-\left(\text { VPGEG) }{ }_{2}\right]_{n}$ LPETGG

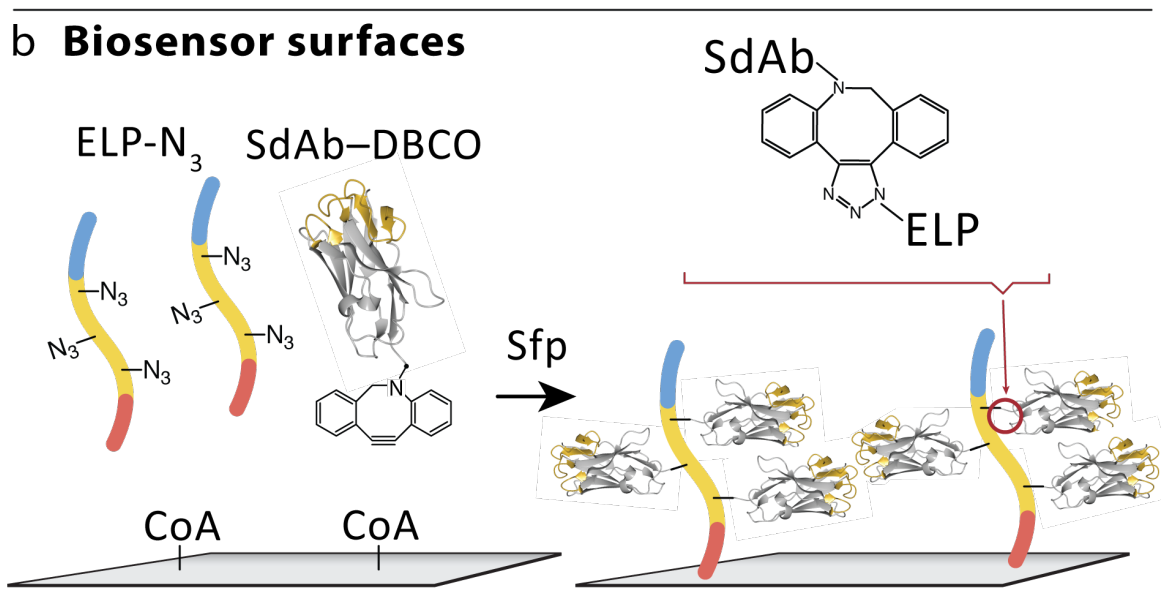

c Fluorescent cytometry

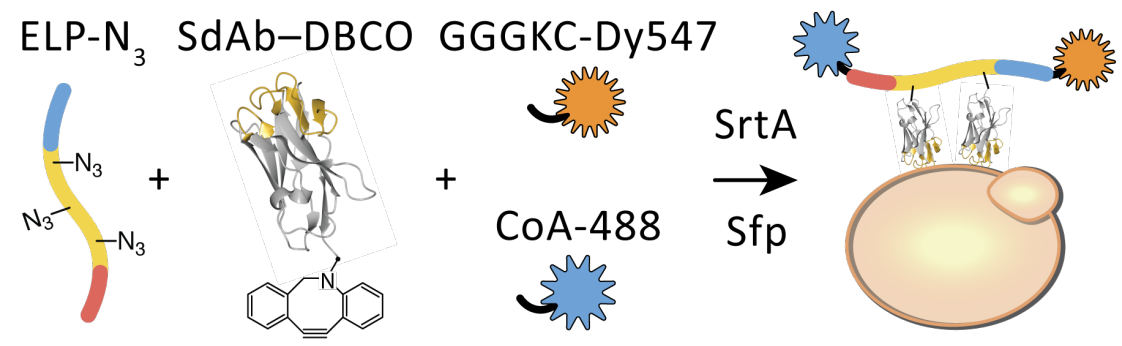

Figure 1. (a) ELP design containing multisite L-azidohomoalanine ( $\left.\mathrm{M}^{\text {azido }}\right)$ guest residues, and terminal ybbR and sortase A tags. (b) One-pot strategy for assembly of an immunoassay surface comprising site-specifically oriented multi-antibody complexes assembled using SPAAC and Sfp-mediated enzyme ligation. (c) Strategy for loading ELP$\mathrm{N}_{3}$ with 3 cargo types using SPAAC, Sfp and SrtA enzyme ligations. The resulting complexes are used to target and label antigen-presenting eukaryotic cells. 
Both wild-type and azide-containing ELPs (denoted as ELP-wt and ELP- $\mathrm{N}_{3}$, respectively) were well expressed in yields of $10-20 \mathrm{mg} / \mathrm{L}$ culture media. Yields for ELP-wt were slightly higher than those for ELP-N $\mathrm{N}_{3}$, and both ELPs were obtained in high purity following ITC based on SDS-PAGE analysis (Fig. 2A). ELP-wt exhibited phase transition behavior in PBS with an LCST of $75-80{ }^{\circ} \mathrm{C}$, whereas ELP-N ${ }_{3}$ did not show any LCST between 4 and $80{ }^{\circ} \mathrm{C}$ when tested in buffer solutions of pH $6.5-8.5$ or in PBS solution lacking additional $\mathrm{NaCl}$ (Fig. S2, Supporting information). When $2 \mathrm{M} \mathrm{NaCl}$ was added, the LCSTs of all ELPs were dramatically reduced down to $25^{\circ} \mathrm{C}$ (Fig. 2B).
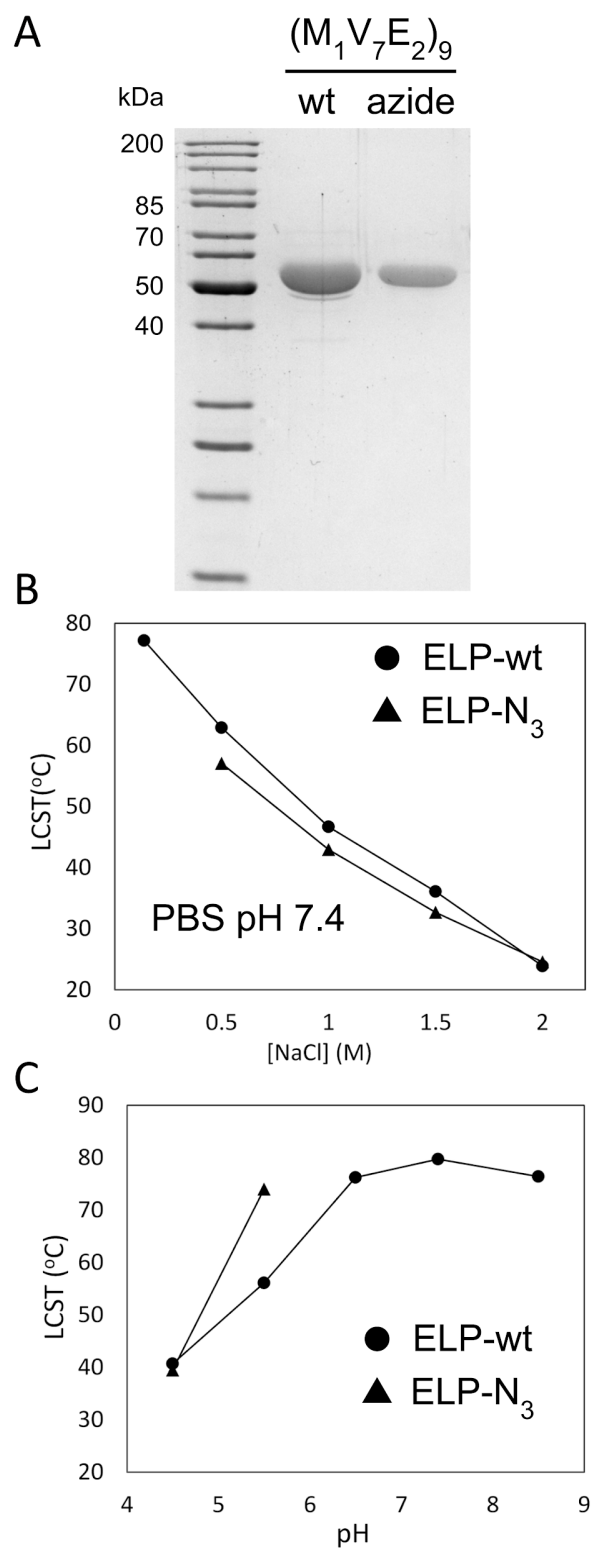

Figure 2. (A) SDS-PAGE of purified ELP-wt and azide-containing ELP-N $\mathrm{N}_{3}$ with 90 VPGXG repeats. (B) lonic strength dependence of the LCST of ELP-wt and ELP-N $\mathrm{N}_{3}$. (C) pH-responsive LCST characteristics of ELP-wt and ELP-N . $_{3}$

The ELP scaffold was designed to accommodate three orthogonal conjugation chemistries: (i) SPAAC between dibenzocyclooctyne (DBCO) groups and multi-site azide groups, (ii) Sfp-mediated ligation via the $\mathrm{N}$-terminal ybbR tag to coenzyme A (CoA) substrates, and (iii) Sortase A-mediated ligation via the Cterminal LPETGG tag to GGG-substrates. To verify the presence and functionality these groups, ELP-N $\mathrm{N}_{3}$ was tested with fluorescent dyes and proteins containing respective complementary groups. DBCO-SulfoCy5 was used to test multi-site SPAAC-mediated conjugation to the azide groups. CoA-AlexaFluor 488 (CoA-488) was used to test reaction with the ybbR tag via Sfp transferase, and a second protein domain (a 
carbohydrate binding module from $C$. thermocellum, CBM) containing an N-terminal GGG-sequence was used to test Sortase A-mediated protein ligation ${ }^{46}$.

ELP-wt and ELP-N ${ }_{3}$ both contained the ybbR tag, and as expected both could be conjugated with CoA-488 (Fig. 3A) in the presence of Sfp. Similarly, both ELP-wt and ELP-N ${ }_{3}$ scaffolds could be successfully ligated to GGG-CBM (Fig. 3B) by sortase A-mediated ligation. However, the DBCO-Sulfo-Cy5 was successfully conjugated only to ELP-N $\mathrm{N}_{3}$ and not ELP-wt due to the specificity of SPAAC which required the azide groups on ELP-N 3 .

We first estimated the number of successful azidohomoalanine replacements in the ELP by spectrophotometric analysis following reaction with DBCO-Sulfo-Cy5. Fig. 3C shows the SEC chromatogram of the reaction mixture from which the degree of labeling (DOL) could be estimated (see methods). The analysis showed that following conjugation there were $\sim 8.8$ dye molecules per ELP-N $\mathrm{N}_{3}$, with a theoretical value of 9 corresponding to $100 \%$ replacement of methionine with azide groups and stoichiometric modification by DBCO-Sulfo-Cy5. This result indicated that sense suppression and multi-site azide incorporation was highly efficient in the RF11 E. coli strain, approaching $100 \%$, and that these residues were highly reactive toward DBCO. MALDI-TOF mass spectrometry (Fig. 3D and E) showed that the molecular weight (MW) of ELP-N 3 was $\sim 46 \mathrm{Da}$ lower than that of the ELP-wt. Since the MW of azidohomoalanine $(144.13 \mathrm{~g} / \mathrm{mol})$ is $5 \mathrm{~g} / \mathrm{mol}$ less than that of canonical methionine $(149.21 \mathrm{Da})$, we attributed the decrease of $\sim 46 \mathrm{Da}$ to the incorporation of $\sim 9$ azidohomoalanine residues into each ELP chain. The fact that the obtained mass of both ELP-wt and ELP-N $\mathrm{N}_{3}$ were $\sim 206 \mathrm{Da}$ higher than the theoretically calculated values was attributed to the attachment of sinapinic acid adducts from the MALDI matrix to lysine residues ${ }^{43}$ present in the ybbR tag.

A

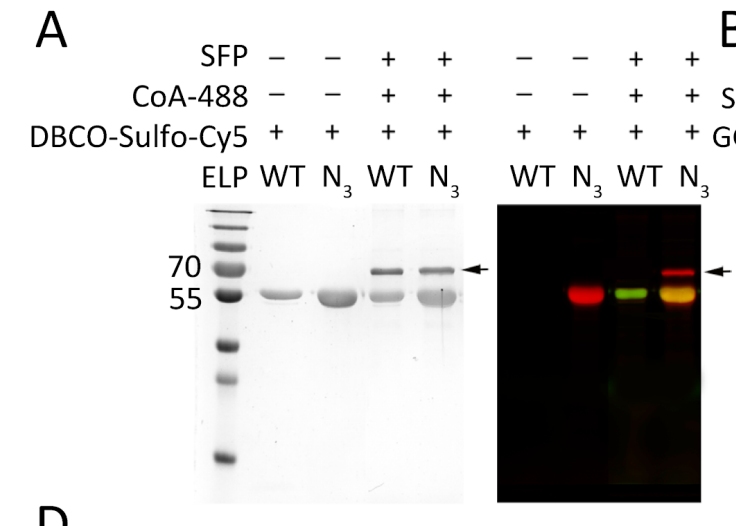

B

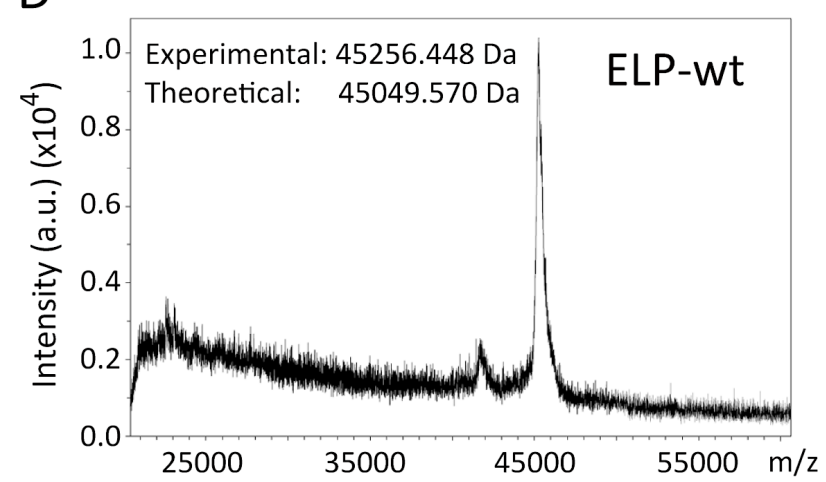

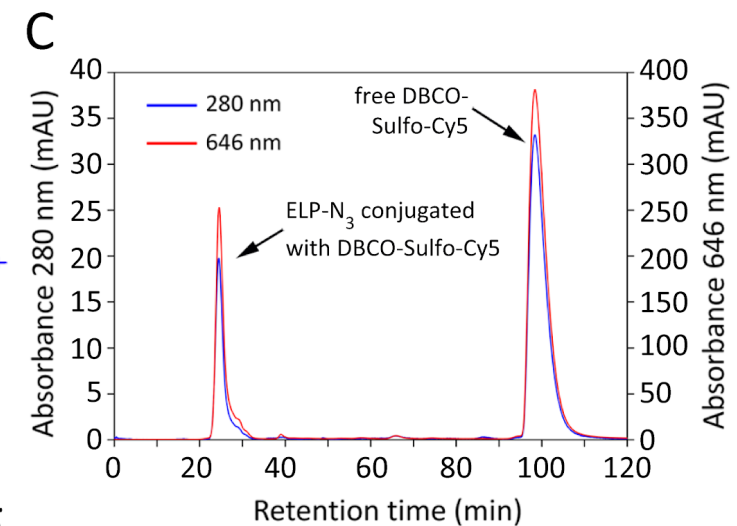

E

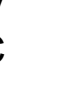


To impart specific bioaffinity to the ELP scaffolds, we next sought to functionalize the 9 azide groups on ELP- $\mathrm{N}_{3}$ with SdAbs targeting the fluorescent protein mCherry. Since the SdAb binds via its N-terminal region, we engineered a C-terminal cysteine into the SdAb sequence at the DNA level and expressed and purified the protein from $E$. coli. The C-terminal cysteine was then modified with a DBCO functional group via thiol-maleimide coupling (see supporting methods).

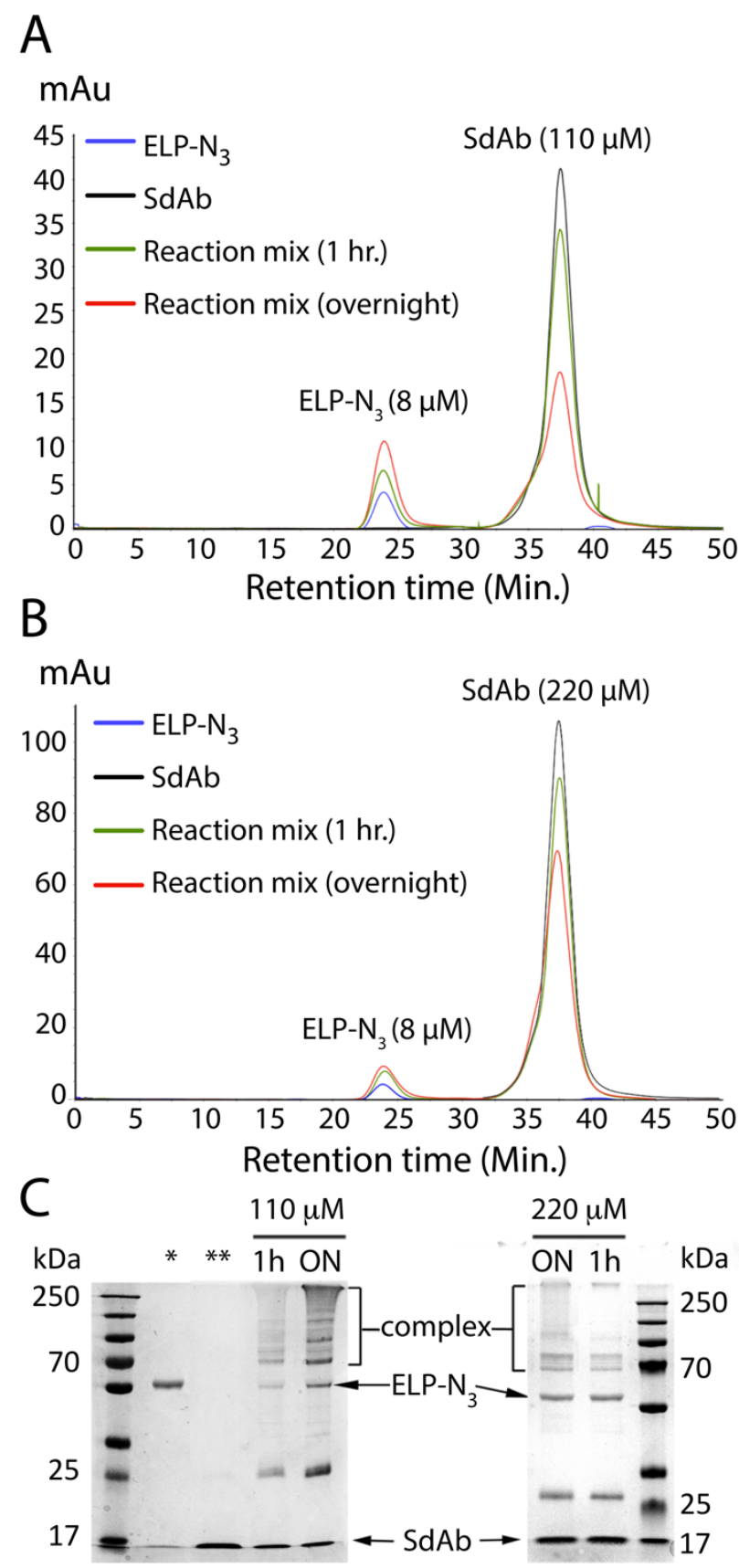

Figure 4. SEC analysis of the conjugation reaction between DBCO-functionalized SdAb and ELP-N $\mathrm{N}_{3}$ scaffolds at (A) $110 \mu \mathrm{M}$ or (B) $220 \mu \mathrm{M}$ of SdAb with ELP-N $\mathrm{N}_{3}$ fixed at $8 \mu \mathrm{M}$ after $1 \mathrm{hr}$ or overnight (ON) incubation. (C) SDS-PAGE analysis for the reaction mix. Lane *: ELP-N 3 only, lane **: SdAb only as controls.

The SPAAC reaction to covalently attach multiple DBCO-modified SdAbs onto ELP-N $\mathrm{N}_{3}$ was performed by simply mixing the two proteins in Tris-buffer at $\mathrm{pH}$ 7.5. The SEC chromatograms (Fig. 4A and B) showed a decrease in free SdAb and an increase in the ELP-N $\mathrm{N}_{3}$ peak after $1 \mathrm{hr}$-conjugation, indicating successful conjugation of SdAbs. Incubating the reaction overnight improved the conjugation efficiency and further decreased the height of the SdAb peak to that of the ELP on SEC chromatogram. The SEC peaks of SdAb- 
modified ELP-N $\mathrm{N}_{3}$ complexes were not shifted toward shorter retention times when compared with free ELP$\mathrm{N}_{3}$ despite the higher molecular weight of the complexes as observed in SDS-PAGE. This result suggested that the intrinsically disordered structure of the ELP scaffold was a determining factor for the retention time on the SEC column, and that linkage to SdAbs did not significantly alter the hydrodynamic size of ELP-N 3 . SDS-PAGE result (Fig. 4C) showed several products with MWs from 70 to $250 \mathrm{kDa}$ after $1 \mathrm{hr}$ of reaction. The band at $25 \mathrm{kDa}$ was attributed to disulfide linked DBCO-SdAb dimers. A mixture of different MW proteins observed in the SDS PAGE indicated that the SdAb-ELP complexes had different numbers of $S d A b$ molecules, with an average of roughly $\sim 4$ SdAbs per ELP chain.

We next demonstrated the use ELP-N $\mathrm{N}_{3}$ for surface modification of microwell plates (Fig. 1B). We performed a sandwich immunoassay for the mCherry antigen and compared the results obtained from surfaces with randomly immobilized SdAbs to surfaces with controlled attachment of SdAbs onto ELP-N 3 scaffolds. The multi-SdAb:ELP complexes or the randomly adsorbed SdAbs served as the primary capture antibodies, while the detection antibody was a horseradish peroxidase-conjugated anti-mCherry monoclonal antibody. Previously, it was shown that site-specific immobilization of SdAbs on biosensor surfaces could improve antigen binding capacity and detection sensitivity, and was preferable over randomly oriented approaches $^{44}$. Our ELP-N $\mathrm{N}_{3}$ scaffold simultaneously achieved controlled orientation as well as an increase in surface binding capacity based on the multivalent properties of the scaffold design.

To build the biosensor surface, a single mixture containing ELP-N $\mathrm{N}_{3}$, DBCO-functionalized SdAb and Sfp was applied to a 96-well maleimide functionalized microwell plate that had been pre-functionalized with CoA groups (Fig. 1b). Overnight incubation resulted in Sfp-mediated covalent site-specific immobilization of the ELP-N $\mathrm{N}_{3}$ scaffold to the surface via the N-terminal ybbR tag, and simultaneous assembly of multiple copies of the DBCO-SdAb onto the ELP-N $\mathrm{N}_{3}$ scaffold. ELP- $\mathrm{N}_{3}: S d A b$ surfaces prepared in this way were compared with surfaces modified by random non-specific adsorption of SdAb molecules at the same total $\mathrm{SdAb}$ concentration as was used for ELP-N $\mathrm{N}_{3}$ surfaces. Fig. $5 \mathrm{~A}$ shows the results of such an immunoassay on mCherry spiked into buffer. The results demonstrate that surfaces prepared with immobilized ELP$\mathrm{N}_{3}: \mathrm{SdAb}$ complexes exhibited a dose-response curve with steeper slope and lower limit of detection than that for the SdAb randomly adsorbed onto the surface. Comparing the two immunoassays in buffer (Table 1 , clean buffer) shows that the multi-SdAb:ELP scaffolds conferred a 13.5-fold increase in detection sensitivity, a 2.35 -fold decrease in apparent $K_{D}$ of the interaction, and a 1.69-fold decrease in the limit of detection.

We next performed a series of immunoassays on mCherry spiked into undiluted human plasma. To test the effect of the maleimide-modified plates, we compared the site-specific multi-antibody ELP complexes with randomly adsorbed antibodies on different multi-well plates. The first plate for the randomly adsorbed antibodies was the same plate as was used for ELP-scaffold immobilization (i.e., maleimide-functionalized plates). We also performed the non-specific adsorption step on Maxisorp plates (Nunc) which are manufactured for immunoassays to non-specifically bind $600-650 \mathrm{ng}$ antibody/ $/ \mathrm{cm}^{2}$. We also tested the effects of DBCO-modification on the non-specific adsorption of SdAbs.

Figure 5B shows the results of the immunoassays of mCherry spiked into undiluted human plasma. The fitted parameters for these immunoassays are presented in Table 1. DBCO-modification of the SdAbs alone improved the non-specific adsorption-based immunoassay performance, likely because of the hydrophobic nature of the DBCO group which enhanced adsorption to Maxisorp. Even when considering the best-performing immunoassay utilizing non-specific adsorption on maxisorp plates, a significant improvement for the multi-SdAb:ELP complexes was clearly observed. The best performing immunoassay utilizing non-specific adsorption was that utilizing SdAb-DBCO adsorbed onto Nunc Maxisorp plates (Figure 5B, purple trace) with a sensitivity of $0.38 \mathrm{mM}^{-1}$ and a limit of detection of $274 \mathrm{nM}$. The surfaces modified with multi-antibody ELPs complexes, by contrast, exhibited a sensitivity of $0.86 \mathrm{mM}^{-1}$ and a limit of 
detection of $172 \mathrm{nM}$. This represented a $126 \%$ increase in sensitivity across the linear range and a $37 \%$ decrease in the limit of detection. The improvement in sensitivity and limit of detection for the surface modified with multi-antibody ELPs is attributed to multivalency effects of the antibodies on the ELP scaffold which increased the overall binding capacity of the surface.
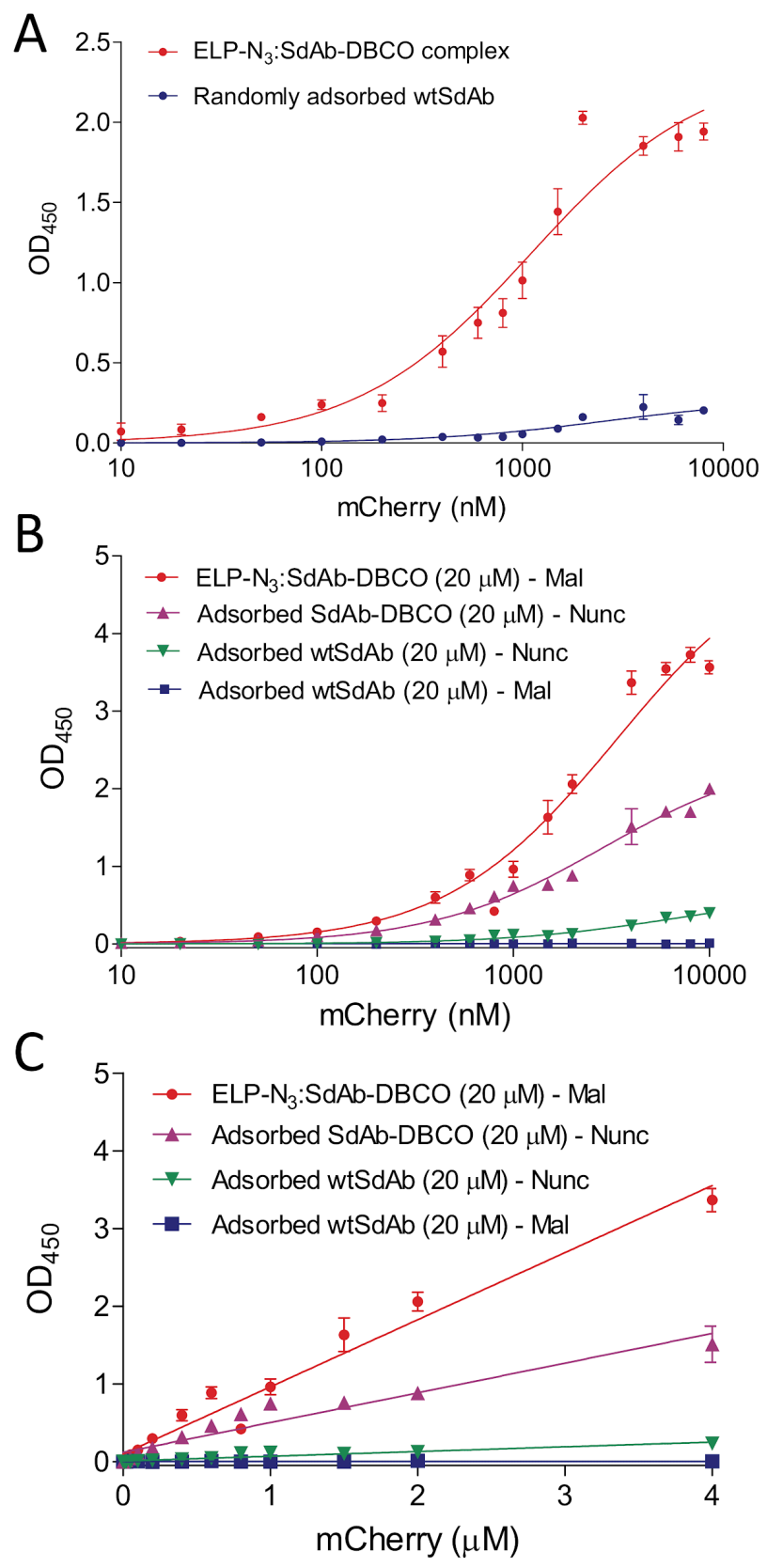

Figure 5. Assembly of SdAbs onto ELP-N $\mathrm{N}_{3}$ for enhancing immunoassay performance (A) Dose-response curves for detection of mCherry spiked into clean buffer using the Sfp-mediated immobilized ELP-N $\mathrm{N}_{3}: \mathrm{SdAb}$ complex or the randomly adsorbed SdAbs onto maleimide-functionalized microplate wells. (B) Dose-response curves for the same immunoassay performed by spiking mCherry into undiluted human plasma. (Mal): maleimide-functionalized microwell plates; (Nunc): Maxisorption immunoassay plates. (C) The linear range of the immunoassay shown in panel B was analyzed to determine the sensitivity. 
Table 1. Fitted parameters and sensitivity of immunoassay dose response curves shown in Figure 5. Mal: maleimide-activated plate; Maxisorp: Nunc-Maxisorp immunoassay plate; ND: not detected

\begin{tabular}{|c|c|c|c|c|c|}
\hline Matrix & Primary capture antibody & Plate & $\mathrm{K}_{\mathrm{D}}(\mu \mathrm{M})$ & Sensitivity $\left(\mathrm{mM}^{-1}\right)$ & $\begin{array}{l}\text { Detection } \\
\text { limit }(n M)\end{array}$ \\
\hline \multirow[t]{2}{*}{$\begin{array}{l}\text { Clean } \\
\text { buffer }\end{array}$} & $\begin{array}{l}\text { ELP-N } \mathrm{N}_{3}: \mathrm{SdAb}-\mathrm{DBCO} \\
\text { complex }\end{array}$ & Mal & $1.10 \pm 0.15$ & $0.95 \pm 0.03$ & $97 \pm 14$ \\
\hline & $\begin{array}{l}\text { Randomly } \quad \text { adsorbed } \\
\text { SdAb-WT }\end{array}$ & Mal & $2.59 \pm 0.94$ & $0.07 \pm 0.01$ & $164 \pm 47$ \\
\hline \multirow[t]{4}{*}{ Plasma } & $\begin{array}{l}\text { ELP-N }{ }_{3}: S d A b-D B C O \\
\text { complex }\end{array}$ & Mal & $3.35 \pm 0.42$ & $0.86 \pm 0.03$ & $172 \pm 27$ \\
\hline & $\begin{array}{l}\text { Randomly } \quad \text { adsorbed } \\
\text { SdAb-WT }\end{array}$ & Mal & $6.75 \pm 0.03$ & ND & $6000 \pm 1786$ \\
\hline & $\begin{array}{l}\text { Randomly } \quad \text { adsorbed } \\
\text { SdAb-WT }\end{array}$ & Maxisorp & $6.91 \pm 0.81$ & $0.06 \pm 0.01$ & $248 \pm 68$ \\
\hline & $\begin{array}{l}\text { Randomly } \quad \text { adsorbed } \\
\text { SdAb-DBCO }\end{array}$ & Maxisorp & $2.83 \pm 0.30$ & $0.38 \pm 0.02$ & $274 \pm 56$ \\
\hline
\end{tabular}

Finally, we used the multi-antibody ELP scaffolds for immunospecific labelling of antigen-presenting cells. We simultaneously conjugated the ELP scaffolds with 3 components: (1) SdAbs, (2) a fluorescent dye CoA488, and (3) the labeled peptide GGGYKC-Dylight 547 (Dy547). The fluorescent molecules were conjugated site-specifically at the $\mathrm{N}$ - and the $\mathrm{C}$ - terminus by Sfp- and sortase A-mediated ligation, respectively. The fluorescent multi-antibody complex was purified by SEC (Fig. 6A). SEC data showed a peak corresponding to the fully assembled complex containing CoA-488, the labelled GGG-peptide, the ELP scaffold and multiple SdAbs. SDS-PAGE analysis of the mixture confirmed successful incorporation of multiple SdAbs and the two fluorescent dyes into the scaffold (Fig. 6A inset). The absorbance spectrum of the purified complex is shown in Fig. 6B. The estimated DOLs of both dye molecules was $\sim 100 \%$ based on an A280 absorption assumption that on average 4 SdAbs were conjugated to each ELP scaffold.

The purified complex was then incubated with two cell populations, one displaying mCherry on the outer cell wall (see Fig. S5, Supporting information for the display efficiency) and the second lacking mCherry (negative control). Fig. 6C shows that the complex specifically bound the mCherry-positive cells, causing an increase in green fluorescence, whereas the negative control population remained unlabeled. The histograms (Fig. 6D) indicated a shift of the population in the green fluorescence channel due to the bound complex which contained CoA-488 dye. The conjugated Dy547 also triggered an increase in the red fluorescence channel. These multivalent SdAb complex can enhance the apparent binding strength of the antibody to the target cells based on avidity effects of the multivalent SdAb carrier, analogous to the results from the plate-based immunoassay. 


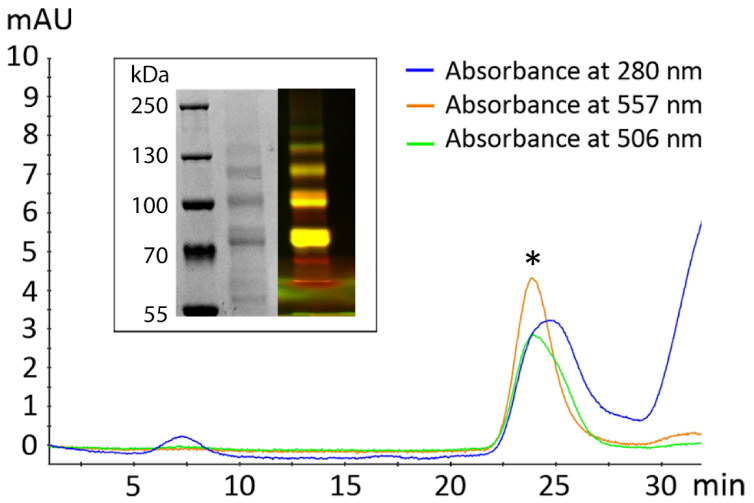

C
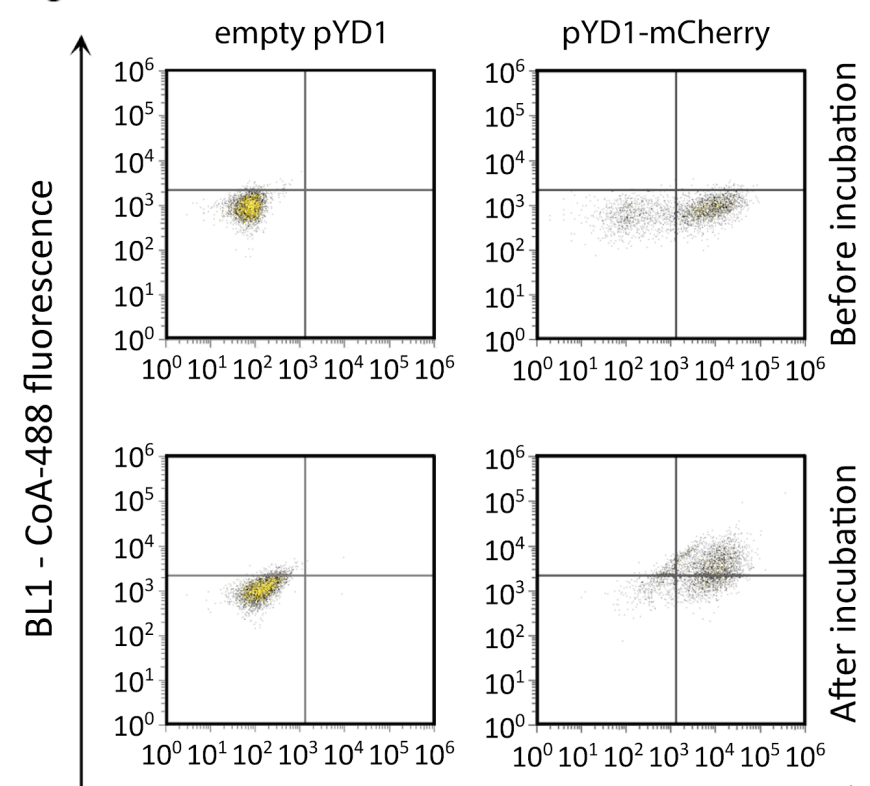

YL2 - mCherry fluorescence
B

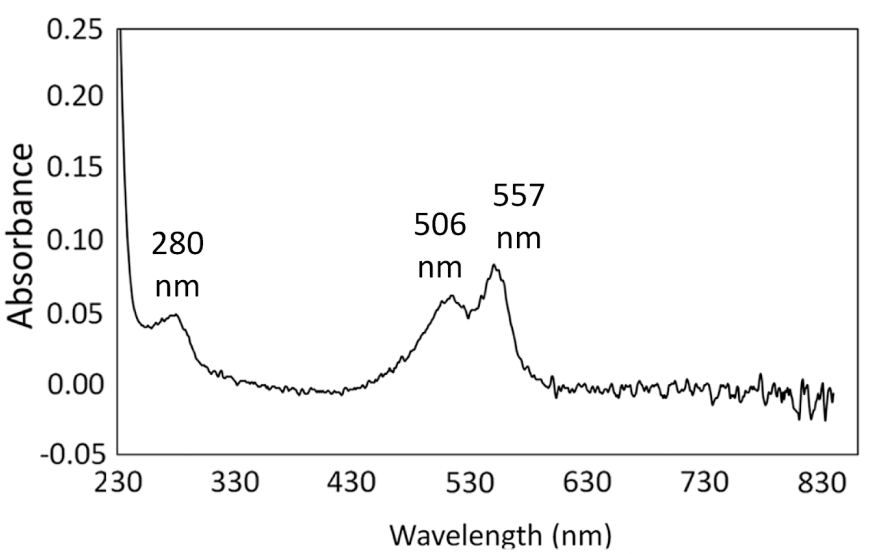

D

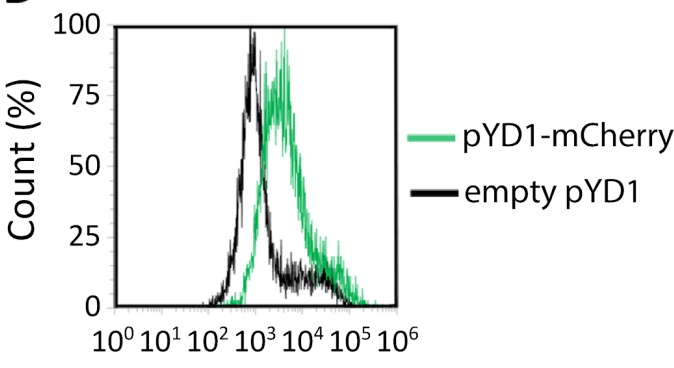

BL1 - CoA-488 fluorescence

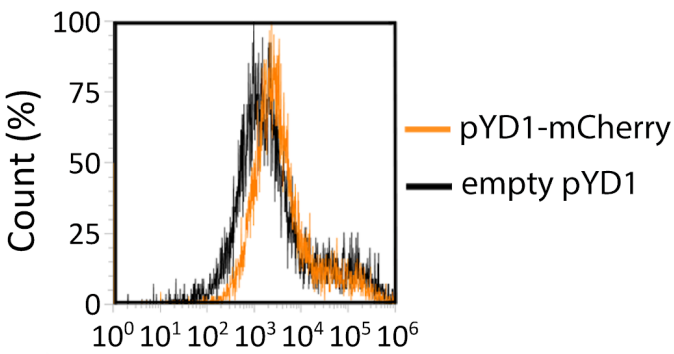

YL2 - mCherry fluorescence

Figure 6. (A) SEC purification of the assembled CoA-488/GGGYKC-Dy547/ELP-N 3 :SdAb complex (*) with two fluorescent dyes at the $\mathrm{N}$ - and the C-terminus. Inset: SDS-PAGE analysis and fluorescent visualization of the complex after SEC, the (B) Light absorbance spectrum of the complex showing three peaks at $280 \mathrm{~nm}$ (the protein), $506 \mathrm{~nm}$ (the CoA-488 dye) and $557 \mathrm{~nm}$ (the Dy547 dye). (C) Flow-cytometric analysis of the binding of the assembled complex to yeast cells carrying empty pYD1 plasmid (no mCherry display) and ones carrying pYD1-mCherry plasmid (with mCherry display). (D) Histograms showing cell counts (in percentage) regarding to their fluorescent signals.

\section{Conclusions}

In summary, we presented a new approach for non-standard guest residue incorporation into ELPs, and for assembling multi-antibody complexes onto the bioorthogonal ELPs as scaffold molecules. We demonstrate that these multi-antibody ELP complexes are able to significantly improve the performance of immunospecific biosensors and achieve specific multi-channel cell labeling. Our sense suppression approach for non-standard guest residue engineering required only a Met-auxotrophic E. coli strain and a single coding plasmid, thereby simplifying the system compared to Amber suppression approaches. Our design comprised periodically occurring Met codons in the ELP guest residue position along with two enzymatic tags (ybbR and sortase A) at the termini of the ELP. All of the assembly sites are genetically encoded, providing precision and versatility in designing the spacing and valency of the resulting multiprotein complexes. The three reaction chemistries used here are bioorthogonal and mutually 
compatible, allowing for one-pot assembly of macromolecular structures and opening the door to in vivo assembly reactions. We utilized this material for the assembly of multi-antibody complexes linked together with various terminal cargo molecules such as CoA-dye molecules, GGG-containing protein domains and labeled GGG peptides onto an ELP scaffold. These examples highlight the potential of such an approach as a generalizable assembly strategy for multiprotein complexes in biomolecular systems.

\section{Supporting Information}

Materials and experimental methods.

\section{Funding Sources}

The authors acknowledge funding from the National Centre for Competence in Research: Molecular Systems Engineering (NCCR-MSE), from the Human Frontier Science Program (HFSP) Young Investigator Grant (RGY80/2015), and from an ERC Starting Grant (MMA 715207).

\section{References}

(1) Banerjee, A.; Howarth, M. Nanoteamwork: Covalent Protein Assembly beyond Duets towards Protein Ensembles and Orchestras. Curr. Opin. Biotechnol. 2017, 51, 16-23.

(2) Horn, A. H. C.; Sticht, H. Synthetic Protein Scaffolds Based on Peptide Motifs and Cognate Adaptor Domains for Improving Metabolic Productivity. Front Bioeng Biotechnol 2015, 3, 191.

(3) Fierobe, H.-P.; Mingardon, F.; Mechaly, A.; Bélaïch, A.; Rincon, M. T.; Pagès, S.; Lamed, R.; Tardif, C.; Bélaïch, J.-P.; Bayer, E. A. Action of Designer Cellulosomes on Homogeneous versus Complex Substrates: Controlled Incorporation of Three Distinct Enzymes into a Defined Trifunctional Scaffoldin. J. Biol. Chem. 2005, 280 (16), 16325-16334.

(4) Heyman, A.; Barak, Y.; Caspi, J.; Wilson, D. B.; Altman, A.; Bayer, E. A.; Shoseyov, O. Multiple Display of Catalytic Modules on a Protein Scaffold: Nano-Fabrication of Enzyme Particles. J. Biotechnol. 2007, 131 (4), 433-439.

(5) Moraïs, S.; Barak, Y.; Caspi, J.; Hadar, Y.; Lamed, R.; Shoham, Y.; Wilson, D. B.; Bayer, E. A. Cellulase-Xylanase Synergy in Designer Cellulosomes for Enhanced Degradation of a Complex Cellulosic Substrate. MBio 2010, 1 (5), e00285-10.

(6) Dueber, J. E.; Wu, G. C.; Malmirchegini, G. R.; Moon, T. S.; Petzold, C. J.; Ullal, A. V.; Prather, K. L. J.; Keasling, J. D. Synthetic Protein Scaffolds Provide Modular Control over Metabolic Flux. Nat. Biotechnol. 2009, 27 (8), 753-759.

(7) Schoffelen, S.; van Hest, J. C. M. Chemical Approaches for the Construction of Multi-Enzyme Reaction Systems. Curr. Opin. Struct. Biol. 2013, 23 (4), 613-621.

(8) Bashor, C. J.; Helman, N. C.; Yan, S.; Lim, W. A. Using Engineered Scaffold Interactions to Reshape MAP Kinase Pathway Signaling Dynamics. Science 2008, 319 (5869), 1539-1543.

(9) You, C.; Zhang, Y. H. P. Self-Assembly of Synthetic Metabolons through Synthetic Protein Scaffolds: One-Step Purification, Co-Immobilization, and Substrate Channeling. ACS Synth. Biol. 2013, 2 (2), 102-110.

(10) Sun, Q.; Madan, B.; Tsai, S.-L.; DeLisa, M. P.; Chen, W. Creation of Artificial Cellulosomes on DNA Scaffolds by Zinc Finger Protein-Guided Assembly for Efficient Cellulose Hydrolysis. Chem. Commun. 2014, 50 (12), 1423-1425.

(11) Wang, Z.-G.; Ding, B. Engineering DNA Self-Assemblies as Templates for Functional Nanostructures. Acc. Chem. Res. 2014, 47 (6), 1654-1662.

(12) Simmel, F. C. DNA-Based Assembly Lines and Nanofactories. Curr. Opin. Biotechnol. 2012, 23 (4), $516-521$.

(13) Chen, R. P.; Blackstock, D.; Sun, Q.; Chen, W. Dynamic Protein Assembly by Programmable DNA Strand Displacement. Nat. Chem. 2018, 10, 474-481.

(14) Koehler, C.; Sauter, P. F.; Wawryszyn, M.; Girona, G. E.; Gupta, K.; Landry, J. J. M.; Fritz, M. H.-Y.; Radic, K.; Hoffmann, J.-E.; Chen, Z. A.; Zou, J.; Tan, P. S.; Galik, B; Junttila, S.; Stolt-Bergner, P.; Pruneri, G.; Gyenesei, A.; Schultz, C.; Biskup, M. B.; Besir, H.; Benes, V.; Rappsilber, J.; Jechlinger, M.; Korbel, J. O.; Berger, I.; Braese, S.; Lemke, E. A. Genetic Code Expansion for Multiprotein Complex Engineering. Nat. Methods 2016, 13 (12), 997-1000. 
(15) Ott, W.; Nicolaus, T.; Gaub, H. E.; Nash, M. A. Sequence-Independent Cloning and PostTranslational Modification of Repetitive Protein Polymers through Sortase and Sfp-Mediated Enzymatic Ligation. Biomacromolecules 2016, 17 (4), 1330-1338.

(16) Fierer, J. O.; Veggiani, G.; Howarth, M. SpyLigase Peptide-Peptide Ligation Polymerizes Affibodies to Enhance Magnetic Cancer Cell Capture. Proc. Natl. Acad. Sci. U. S. A. 2014, 111 (13), E1176E1181.

(17) Veggiani, G.; Nakamura, T.; Brenner, M. D.; Gayet, R. V.; Yan, J.; Robinson, C. V.; Howarth, M. Programmable Polyproteams Built Using Twin Peptide Superglues. Proc. Natl. Acad. Sci. U. S. A. 2016, 113 (5), 1202-1207.

(18) Bellucci, J. J.; Bhattacharyya, J.; Chilkoti, A. A Noncanonical Function of Sortase Enables SiteSpecific Conjugation of Small Molecules to Lysine Residues in Proteins. Angew. Chem. Int. Ed. 2014.

(19) Ghoorchian, A.; Cole, J. T.; Holland, N. B. Thermoreversible Micelle Formation Using a ThreeArmed Star Elastin-like Polypeptide. Macromolecules 2010, 43 (9), 4340-4345.

(20) Ta, D. T.; Vanella, R.; Nash, M. A. Magnetic Separation of Elastin-like Polypeptide Receptors for Enrichment of Cellular and Molecular Targets. Nano Lett. 2017.

(21) Garanger, E.; Lecommandoux, S. Towards Bioactive Nanovehicles Based on Protein Polymers. Angew. Chem. Int. Ed Engl. 2012, 51 (13), 3060-3062.

(22) Bellucci, J. J.; Amiram, M.; Bhattacharyya, J.; McCafferty, D.; Chilkoti, A. Three-in-One Chromatography-Free Purification, Tag Removal, and Site-Specific Modification of Recombinant Fusion Proteins Using Sortase A and Elastin-like Polypeptides. Angew. Chem. Int. Ed Engl. 2013, 52 (13), 3703-3708.

(23) Ott, W.; Jobst, M. A.; Bauer, M. S.; Durner, E.; Milles, L. F.; Nash, M. A.; Gaub, H. E. Elastin-like Polypeptide Linkers for Single-Molecule Force Spectroscopy. ACS Nano 2017, 11 (6), 6346-6354.

(24) Roberts, S.; Dzuricky, M.; Chilkoti, A. Elastin-like Polypeptides as Models of Intrinsically Disordered Proteins. FEBS Lett. 2015, 589 (19 Pt A), 2477-2486.

(25) McDaniel, J. R.; Bhattacharyya, J.; Vargo, K. B.; Hassouneh, W.; Hammer, D. A.; Chilkoti, A. SelfAssembly of Thermally Responsive Nanoparticles of a Genetically Encoded Peptide Polymer by Drug Conjugation. Angew. Chem. Int. Ed. 2012, 52 (6), 1683-1687.

(26) Smits, F. C. M.; Castelijns, W. W. A.; van Hest, J. C. M. Crosslinked ELP-Based Nanoparticles, Using the Strain Promoted Azide-alkyne Cycloaddition. Eur. Polym. J. 2015/1, 62, 386-393.

(27) Link, A. J.; Mock, M. L.; Tirrell, D. A. Non-Canonical Amino Acids in Protein Engineering. Curr. Opin. Biotechnol. 2003, 14 (6), 603-609.

(28) Lemke, E. A. The Exploding Genetic Code. Chembiochem 2014, 15 (12), 1691-1694.

(29) Chin, J. W. Expanding and Reprogramming the Genetic Code of Cells and Animals. Annu. Rev. Biochem. 2014, 83, 379-408.

(30) Lang, K.; Chin, J. W. Bioorthogonal Reactions for Labeling Proteins. ACS Chem. Biol. 2014, 9 (1), $16-20$.

(31) Plass, T.; Milles, S.; Koehler, C.; Schultz, C.; Lemke, E. A. Genetically Encoded Copper-Free Click Chemistry. Angew. Chem. Int. Ed Engl. 2011, 50 (17), 3878-3881.

(32) Pang, Y.; Liu, J.; Qi, Y.; Li, X.; Chilkoti, A. A Modular Method for the High-Yield Synthesis of SiteSpecific Protein-Polymer Therapeutics. Angew. Chem. Int. Ed Engl. 2016, 55 (35), 10296-10300.

(33) Teeuwen, R. L. M.; van Berkel, S. S.; van Dulmen, T. H. H.; Schoffelen, S.; Meeuwissen, S. A.; Zuilhof, H.; de Wolf, F. A.; van Hest, J. C. M. "Clickable" Elastins: Elastin-like Polypeptides

Functionalized with Azide or Alkyne Groups. Chem. Commun. 2009, 0 (27), 4022-4024.

(34) Wu, I.-L.; Patterson, M. A.; Carpenter Desai, H. E.; Mehl, R. A.; Giorgi, G.; Conticello, V. P. Multiple Site-Selective Insertions of Noncanonical Amino Acids into Sequence-Repetitive Polypeptides.

Chembiochem 2013, 14 (8), 968-978.

(35) Costa, S. A.; Simon, J. R.; Amiram, M.; Tang, L.; Zauscher, S.; Brustad, E. M.; Isaacs, F. J.; Chilkoti, A. Photo-Crosslinkable Unnatural Amino Acids Enable Facile Synthesis of Thermoresponsive Nano- to Microgels of Intrinsically Disordered Polypeptides. Adv. Mater. 2017, 30, 1704878.

(36) Amiram, M.; Haimovich, A. D.; Fan, C.; Wang, Y.-S.; Aerni, H.-R.; Ntai, I.; Moonan, D. W.; Ma, N. J.; Rovner, A. J.; Hong, S. H.; Kelleher, N. L.; Goodman, A. L.; Jewett, M. C.; Söll, D.; Rinehart, J.; Isaacs, F. J. Evolution of Translation Machinery in Recoded Bacteria Enables Multi-Site Incorporation of Nonstandard Amino Acids. Nat. Biotechnol. 2015, 33 (12), 1272-1279.

(37) Zhang, K.; Diehl, M. R.; Tirrell, D. A. Artificial Polypeptide Scaffold for Protein Immobilization. J. Am. Chem. Soc. 2005, 127 (29), 10136-10137.

(38) Nowatzki, P. J.; Franck, C.; Maskarinec, S. A.; Ravichandran, G.; Tirrell, D. A. Mechanically Tunable Thin Films of Photosensitive Artificial Proteins: Preparation and Characterization by Nanoindentation. 
Macromolecules 2008, 41 (5), 1839-1845.

(39) Link, A. J.; Tirrell, D. A. Reassignment of Sense Codons in Vivo. Methods 2005, 36 (3), 291-298.

(40) Schoffelen, S.; Lambermon, M. H. L.; van Eldijk, M. B.; van Hest, J. C. M. Site-Specific Modification of Candida Antarctica Lipase B via Residue-Specific Incorporation of a Non-Canonical Amino Acid. Bioconjug. Chem. 2008, 19 (6), 1127-1131.

(41) Meyer and Ashutosh, D. Genetically Encoded Synthesis of Protein-Based Polymers withPrecisely Specified Molecular Weight and Sequence byRecursive Directional Ligation: Examples from the Elastin-likePolypeptide System. Biomacromolecules 2002, 3, 357-367.

(42) Iwasaki, T.; Fukazawa, R.; Miyajima-Nakano, Y.; Baldansuren, A.; Matsushita, S.; Lin, M. T.; Gennis, R. B.; Hasegawa, K.; Kumasaka, T.; Dikanov, S. A. Dissection of Hydrogen Bond Interaction Network around an Iron-Sulfur Cluster by Site-Specific Isotope Labeling of Hyperthermophilic Archaeal Rieske-Type Ferredoxin. J. Am. Chem. Soc. 2012, 134 (48), 19731-19738.

(43) Fagerquist, C. K.; Sultan, O.; Carter, M. Q. Possible Evidence of Amide Bond Formation between Sinapinic Acid and Lysine-Containing Bacterial Proteins by Matrix-Assisted Laser Desorption/ionization (MALDI) at 355 Nm. J. Am. Soc. Mass Spectrom. 2012, 23 (12), 2102-2114.

(44) Ta, D. T.; Guedens, W.; Vranken, T.; Vanschoenbeek, K.; Steen Redeker, E.; Michiels, L.; Adriaensens, P. Enhanced Biosensor Platforms for Detecting the Atherosclerotic Biomarker VCAM1 Based on Bioconjugation with Uniformly Oriented VCAM1-Targeting Nanobodies. Biosensors 2016, 6 (3), 34.

(45) Petitdemange, R.; Garanger, E.; Bataille, L.; Bathany, K.; Garbay, B.; Deming, T. J.; Lecommandoux, S. Tuning Thermoresponsive Properties of Cationic Elastin-like Polypeptides by Varying Counterions and Side-Chains. Bioconj. Chem. 2017, 28 (5), 1403-1412.

(46) Liu, H.; Ta, D. T.; Nash, M. A. Mechanical Polyprotein Assembly using Sfp and Sortase-mediated Domain Oligomerization for Single-molecule Studies. Small Methods 2018, 2 (6), 1800039.

\section{Table of Contents Graphic}

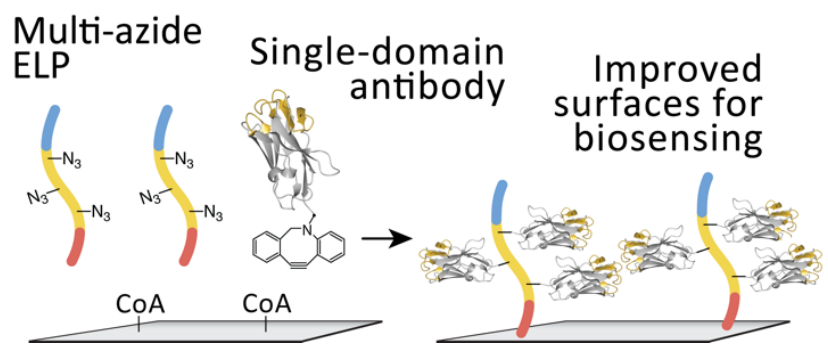

